\begin{tabular}{ccc}
\hline AECORDS OF PHARMACEUTICAL \\
AND BIOMEDICAL SCIENCES
\end{tabular}

\title{
Investigating Pirfenidone and Vitamin D for Targeting Cardiac and Renal Fibrotic Pathways in Experimentally-Induced Animal Model
}

\author{
Mona F. El Azab ${ }^{a}$, Mohamed A. Saleh ${ }^{b}$, Reem M. Hazem ${ }^{a}$, Samar A. Antar $^{c^{*}}$ \\ ${ }^{a}$ Department of Pharmacology \& toxicology, Faculty of Pharmacy, Suez Canal University. Egypt. \\ ${ }^{b}$ Department of Pharmacology \& toxicology, Faculty of Pharmacy, Mansoura University, Mansoura, Egypt. \\ ${ }^{c}$ Department of Pharmacology, Horus university, Damietta, Egypt
}

Received on: 26.10. 2018

Revised on: 20. 12. 2018

Accepted on: 25. 12. 2018

Correspondence Author:

Tel:+ 01010292517

E-mail address:

samarantar38@yahoo.com

\begin{abstract}
Breast cancer is considered as the most familiar cancer in females which represented $38.8 \%$ in Egypt and 29\% in the world. It is the second common cause of cancer-related death in women. Treatment of breast cancer with Doxorubicin may lead to many side effects, mainly cardiac and renal fibrosis. The present study aimed to investigate the underlying molecular mechanisms for the potential anti-fibrotic effect of pirfenidone $(500 \mathrm{mg} / \mathrm{kg}$, P.O. once daily) and Vitamin D $(0.5 \mu \mathrm{g} / \mathrm{kg}$ I.P. once daily) against doxorubicin $(15 \mathrm{mg} / \mathrm{kg}$ I.P.) induced cardio- and renal- fibrosis. Moreover, the anti-cancer potential of pirfenidone (PFD) and Vitamin D either alone or in combination with doxorubicin will be assessed in a xenograft experimental model of breast cancer. Then, tissue and blood samples will be collected after two weeks posttreatment to assess the toxicity of Doxorubicin.
\end{abstract}

Keywords: Breast cancer, Doxorubicin, Pirfenidone, Vitamin D.

\section{Introduction}

Breast cancer is one of the most common lifethreatening diseases among women originating from breast tissue (Gaafer 2015). Treatment of breast cancer includes surgery followed by chemotherapy however; treatment with chemotherapeutic agents may lead to fibrosis in various organs. The development of fibrosis is a dynamic, multifactorial process. This process is initiated by healing after injury and by pathological conditions in which the normal balance of Genesis and breakdown of extracellular matrix (ECM) proteins is disturbed. Fibrogenesis involves the participation of various acute and chronic inflammatory cells that synthesize and secrete profibrotic growth factors. Myofibroblasts and macrophages are 2 key chronic inflammatory cell types that increase in number during fibrosis (Ricardo, Van Goor et al. 2008).

Excessive accumulation of ECM, mostly observed in kidney diseases may lead to renal fibrosis. The pathogenesis of fibrosis is a progressive process that ultimately lead to end-stage renal failure. Simply, renal fibrosis represents a failed wound healing process of the kidney tissue after chronic and sustained injury (Liu 2006). It is characterized by glomerulosclerosis and tubulointerstitial fibrosis expressed as final common manifestation of a wide variety of chronic kidney disease (CKD). The development of renal fibrosis is an unwanted side effect of treatment with chemotherapy (Eitner and Floege 2003).

Cardiac fibrosis is also a common adverse effect associated with cancer treatment, whether it 
is systemic from chemotherapy or immunotherapy or local from radiotherapy. It limits the use of chemotherapeutic agents and is debilitating and lifethreatening for patients(Carvalho, Burgeiro et al. 2014).

In this context, understanding cellular and molecular mechanisms of fibrosis became a necessity. This isn't only for illustrating the complicated pathogenesis of renal and cardiac fibrosis but also, for exploring and validating the efficient anti-fibrotic therapies.

Doxorubicin is anthracycline-based antibiotic that has a widespread application in cancer chemotherapy and used to treat several solid tumors, acute leukemia and malignant lymphoma. (López-Novoa, Martínez-Salgado et al. 2010). Pirfenidone is an anti-fibrotic drug which exhibits well-documented antifibrotic and anti-inflammatory activities in a variety of animal and cell-based models (Prud'Homme 2007). Furthermore, pirfenidone disrupts tumor-stromal interactions by suppressing the synthesis and secretion of factors involved in these interactions like Platelet derived growth factor-A ,collagen type I, and fibronectin (Kozono, Ohuchida et al. 2013). Vitamin D has been reported to have a role in the modulation of renal inflammation in addition to, anticancer activities against many cancer types, including breast cancer (Deeb, Trump et al. 2007).

\section{Review of literature}

Breast cancer is the most common disease that develops from breast tissue. Signs of breast cancer may include a lump in the breast, a change in breast shape, dimpling of the skin, fluid coming from the nipple, a newly inverted nipple, or a red or scaly patch of skin. In those with distant spread of the disease, there may be bone pain, swollen lymph nodes, shortness of breath, or yellow skin (Kabel and Baali 2015). Breast cancer is usually treated with surgery, which may be followed by chemotherapy or radiation therapy, or both. A multidisciplinary approach is the best (Bao and Rudek 2011).

Chemotherapy is predominantly used for cases of breast cancer in stages 2-4 and is particularly beneficial in estrogen receptor-negative (ER-) disease. Treatment with chemotherapeutic agents destroy the physiological homoeostasis and affects multiple organs during treatment process. Chemotherapy is usually administered in combinations, for periods of 3-6 months. Most chemotherapy medications work by eradicating fast-growing and/or fast-replicating cancer cells by causing DNA damage upon replication mechanisms. However, the medications also damage fast-growing normal cells, which may cause serious side effects like fibrosis (Kumral, Soluk-Tekkeşin et al. 2015).

Cardiac fibrosis may refer to an abnormal thickening of the heart valves due to inappropriate proliferation of cardiac fibroblasts but more commonly refers to the excess deposition of extracellular matrix in the cardiac muscle (Gourdie, Dimmeler et al. 2016). Fibrotic cardiac muscle is stiffer and less compliant and is seen in the progression to heart failure. Fibrocyte cells normally secrete collagen to provide structural support for the heart. The thickening and loss of flexibility eventually may lead to valvular dysfunction and heart failure (Berk, Fujiwara et al. 2007).

There are a variety of causes that trigger fibrotic remodeling of the myocardium as hemodynamic, toxic, metabolic, and immunologic disturbances. Among the causes leading to kidney fibrosis are diabetes and hypertension "two principal causes of CKD", infectious glomerulonephritis, renal vasculitis, ureteral obstruction, genetic alterations, autoimmune diseases and drugs like Doxorubicin (LópezNovoa, Martínez-Salgado et al. 2010).

Doxorubicin is an anthracycline commonly used anticancer agent however, severe clinical side effects, such as cardiotoxicity and myelosuppression, are of major concern (Mady 2007). It exerts its antitumor activity through inhibiting the replication process of cells by crossing the tumor cellular membrane and intercalating into the base pairs of DNA. Doxorubicin chemotherapy serves as one of the routines in treating cancer clinically. Doxorubicin, is widely used in the treatment of malignant tumors, such as liver cancer, breast cancer, ovarian cancer, gastric cancer, non-small cell lung cancer, and prostate cancer (Al-Abbasi, Alghamdi et al. 2016). On the other hand, the severe toxic side effects of doxorubicin on human body have restricted its clinical application (Octavia, Tocchetti et al. 2012).

The increased risk of cardiac dysfunction from doxorubicin can manifest acutely during treatment or chronically weeks to years after treatment has 
been ceased. Researches have shown that doxorubicin chemotherapy could generate severe tissue injury in heart and kidney (Kumral, SolukTekkeşin et al. 2015) and the symptoms of renal damage like hematuresis and proteinuria are especially evident (Wang, Wang et al. 2000).

There are various signaling mechanisms involved in doxorubicin cardiotoxicity. First and foremost, the doxorubicin-induced cardiotoxicity is due to oxidative stress. Cardiac mitochondrial damage is supposed after few hours following the exposure to doxorubicin. Cardiac dysfunction may present across a broad spectrum of symptoms that may range from arrhythmias to overt heart failure. Doxorubicin also produced cardiomyopathy due to interstitial and perivascular fibrosis (Aerts, Velazquez et al. 2014). In addition, doxorubicin treatment significantly elevates expression of transforming growth factor-beta (TGF- $\beta$ ) and phosphor-SMAD3 along with increased collagen deposition, an increment of fibroblast to myofibroblast phenotypic transformation and profibrotic signaling pathway(Diwan, Wansapura et al. 2008).

Renal fibrosis is mid to progressive renal failure and end stage renal disease (Liu 2006). One of the mechanisms of renal tissue injury caused by doxorubicin was the free radical production. This results in lipid peroxidation of glomerulus affecting normal physiological function of renal tissues, thus generating metabolic disorders (Heart, Karandrea et al. 2016). Therefore, one of the methods to reduce renal damage is to inhibit peroxidation.

The mechanisms of renal damage caused by doxorubicin chemotherapy mainly consists of two aspects. The first is direct damage in which general toxicity of doxorubicin on cells directly damaged renal cells producing inflammatory response in renal tissues. As a result, inflammatory mediators as TNF- $\alpha$ and interleukin are released, hence strengthening the growth of renal matrix and glomerular sclerosis (Donner, Yeh et al. 2015). The second is indirect damage. Under the action of multiple reductases, doxorubicin produced free radical, inducing lipid peroxidation in glomerular epithelial cell and causing abnormal glucose and protein metabolism. Moreover, elevated urinary albumin was detected due to altering and breaking the structure and filterability of renal filtration membrane (Rashikh, Pillai et al. 2014).

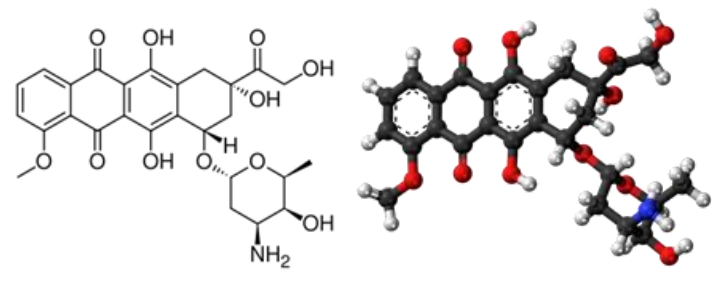

Figure 1: The structure of Doxorubicin.

Pirfenidone is an orally active small molecular comprising a modified phenyl pyridine. Pirfenidone is an anti-fibrotic drug, commonly used for the treatment of idiopathic pulmonary fibrosis which firstly approved in Japan under the trade name Pirespa ${ }^{\circledR}$ and some years later in Europe, United States and Canada under the trade name Esbriet ${ }^{\circledR}$ (Roche Pharmaceuticals) for the same cure. PFD has been reported to exert antiinflammatory, antioxidant, and antifibrotic effects on the heart (Ma, Pan et al. 2014).

Pirfenidone has been shown to reduce the fibrosis of different organs (for example, lung, kidney, liver, heart and vascular remodeling. The compound exhibits well-documented antifibrotic and anti-inflammatory activities in a variety of animal and cell-based models (Schaefer, Ruhrmund et al. 2011). Intraperitoneal and oral administration of PFD reduced the tissue levels of inflammatory markers in both parietal and visceral peritoneum. Also, PFD is an effective agent on the prevention of postoperative fibrosis (Hasdemir, Ozkut et al. 2017)

Pirfenidone (PFD) has been shown to be effective in handling several fibrotic diseases (MacíasBarragán, Sandoval-Rodríguez et al. 2010) by reducing fibroblast proliferation and inhibiting TGF- $\beta$-stimulated collagen production. Furthermore, it can modulate multiple signaling pathways including tumor necrosis factor $\alpha$ (TNF$\alpha$ ), IL-1- $\beta$, and platelet derived growth factor (Kim, Choi et al. 2010). Also, pirfenidone disrupts tumor-stromal interactions by suppressing the synthesis and secretion of factors involved in these interactions like PDGF-A, collagen type I, and fibronectin. Pirfenidone showed antitumor activity (Kozono, Ohuchida et al. 2013). The compound also, exhibits well-documented anti-inflammatory activities in a variety of animal and cell-based models (Schaefer, Ruhrmund et al. 2011). 
pirfenidone disrupts tumor-stromal interactions by suppressing the synthesis and secretion of factors involved in these interactions like PDGF-A, collagen type I, and fibronectin. Pirfenidone suppressed tumor growth, reduced the number of distributed nodules, and reduced the incidence of metastasis. Therefore, combining pirfenidone with traditional anticancer drugs such as gemcitabine may offer a promising treatment strategy for pancreatic cancer (Kozono, Ohuchida et al. 2013).
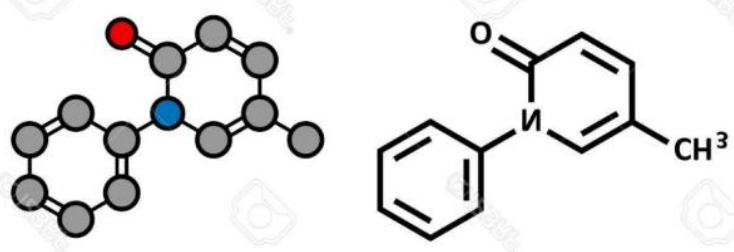

Figure 2. The structure of Pirfenidone

Also, vitamin D is used for treatment of fibrosis. It is a group of fat-soluble steroids responsible for increasing intestinal absorption of calcium, magnesium, and phosphate, and multiple other biological effects. In humans, the most important compounds in this group are vitamin $\mathrm{D}_{3}$ (also known as cholecalciferol) and vitamin $\mathrm{D}_{2}$ (ergocalciferol). Vitamin $\mathrm{D}$ has been reported to have anticancer activities against many cancer types, including breast cancer. In addition, it has been found to have several activities that might slow or prevent the development of cancer, including promoting cellular differentiation, decreasing cancer cell growth, stimulating cell death (apoptosis), and reducing tumor blood vessel formation (Deeb, Trump et al. 2007).

The kidney plays a central role in vitamin D metabolism and regulation of its circulating levels. Therefore, impaired renal function may lead to vitamin D deficiency, as has been observed in patients with CKD (Dusso and Tokumoto 2011). Several studies have shown the role of active vitamin $\mathrm{D}$ in the modulation of renal inflammation (Park, Cho et al. 2012). The anti-inflammatory properties of active vitamin $\mathrm{D}$ and its analogues may be attributed to their ability to suppress the NF- $\mathrm{kB}$ pathway, a key transcription factor that is thought to mediate acute and chronic inflammation and fibrogenesis by regulating gene expression of cytokines, chemokines, and adhesion molecules (including interleukin-6, MCP-1, and tumor necrosis factor- $\alpha$ ) (Guijarro and Egido 2001).
Vitamin D plays a role in protecting against kidney injury by blocking NF- $\mathrm{BB}$ activity and reducing renal inflammation (Schwarz, Amann et al. 1998). In animal models of diabetic nephropathy, treatment with calcitriol and paricalcitol reduces infiltration of inflammatory cells and NF- $\kappa \mathrm{B}$ activation in the glomerulus (Sanchez-Niño, Bozic et al. 2011). Similarly, administration of calcitriol attenuates glomerular hyper cellularity and inflammatory infiltration (Panichi, Migliori et al. 2001).

Studies showed that patients suffering from cardiovascular disease are frequently deficient in the steroid hormone vitamin D (Zittermann, Frisch et al. 2009). Approximately $90 \%$ of chronic HF patients have hypovitaminosis D (Kim, Sabour et al. 2008). Furthermore, vitamin D deficiency might contribute to cardiomyocyte hypertrophy, interstitial inflammation, and fibrosis (Chen, Rosner et al. 2011).Hence, vitamin D deficiency could contribute to a more rapid progression to HF following myocardial damage .
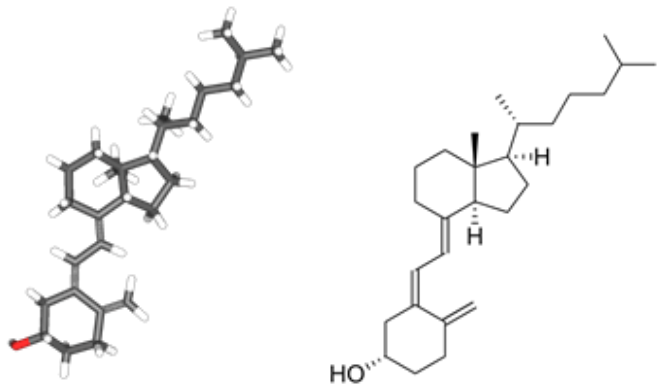

Figure 3. The structure of Vitamin D (Cholecalciferol $\left(\mathbf{D}_{3}\right)$

\section{References}

Aerts, H. J., E. R. Velazquez, R. T. Leijenaar, C. Parmar, P. Grossmann, S. Carvalho, J. Bussink, R. Monshouwer, B. Haibe-Kains and D. Rietveld (2014). "Decoding tumour phenotype by noninvasive imaging using a quantitative radiomics approach." Nature communications 5: 4006.

Al-Abbasi, F. A., E. A. Alghamdi, M. A. Baghdadi, A. J. Alamoudi, A. M. El-Halawany, H. M. ElBassossy, A. H. Aseeri and A. M. Al-Abd (2016). "Gingerol Synergizes the Cytotoxic Effects of Doxorubicin against Liver Cancer Cells and Protects from Its Vascular Toxicity." Molecules 21(7): 886. 
Bao, T. and M. A. Rudek (2011). "The clinical pharmacology of anastrozole." Eur Oncol Haematol 7: 106-108.

Berk, B. C., K. Fujiwara and S. Lehoux (2007). "ECM remodeling in hypertensive heart disease." The Journal of clinical investigation 117(3): 568575.

Carvalho, F. S., A. Burgeiro, R. Garcia, A. J. Moreno, R. A. Carvalho and P. J. Oliveira (2014). "Doxorubicin-induced cardiotoxicity: from bioenergetic failure and cell death to cardiomyopathy." Medicinal research reviews 34(1): 106-135.

Chen, W. Y., B. Rosner, S. E. Hankinson, G. A. Colditz and W. C. Willett (2011). "Moderate alcohol consumption during adult life, drinking patterns, and breast cancer risk." Jama 306(17): 1884-1890.

Deeb, K. K., D. L. Trump and C. S. Johnson (2007). "Vitamin D signalling pathways in cancer: potential for anticancer therapeutics." Nature reviews cancer 7(9): 684.

Diwan, A., J. Wansapura, F. M. Syed, S. J. Matkovich, J. N. Lorenz and G. W. Dorn (2008). "Nix-mediated apoptosis links myocardial fibrosis, cardiac remodeling, and hypertrophy decompensation." Circulation 117(3): 396-404.

Donner, A. J., S. T. Yeh, G. Hung, M. J. Graham, R. M. Crooke and A. E. Mullick (2015). "CD40 generation 2.5 antisense oligonucleotide treatment attenuates doxorubicin-induced nephropathy and kidney inflammation." Molecular Therapy-Nucleic Acids 4.

Dusso, A. S. and M. Tokumoto (2011). "Defective renal maintenance of the vitamin $\mathrm{D}$ endocrine system impairs vitamin $\mathrm{D}$ renoprotection: a downward spiral in kidney disease." Kidney International 79(7): 715-729.

Eitner, F. and J. Floege (2003). "Novel insights into renal fibrosis." Current opinion in nephrology and hypertension 12(3): 227-232.

Gaafer, N. M. (2015). Evaluation of Vitamin D and phosphate levels in serum of breast cancer patients in Khartoum State, Alzaeim Alazhari University.

Gourdie, R. G., S. Dimmeler and P. Kohl (2016). "Novel therapeutic strategies targeting fibroblasts and fibrosis in heart disease." Nature Reviews Drug
Discovery 15(9): 620.

Guijarro, C. and J. Egido (2001). "Transcription factor- $\kappa \mathrm{B}(\mathrm{NF}-\mathrm{\kappa B})$ and renal disease." Kidney International 59(2): 415-424.

Hasdemir, P. S., M. Ozkut, T. Guvenal, M. A. Uner, E. Calik, S. O. Koltan, F. M. Koyuncu and K. Ozbilgin (2017). "Effect of pirfenidone on vascular proliferation, inflammation and fibrosis in an abdominal adhesion rat model." Journal of Investigative Surgery 30(1): 26-32.

Heart, E. A., S. Karandrea, X. Liang, M. E. Balke, P. A. Beringer, E. M. Bobczynski, D. Zayas-Bazán Burgos, T. Richardson and J. P. Gray (2016). "Mechanisms of Doxorubicin Toxicity in Pancreatic $\beta$-Cells." Toxicological Sciences 152(2): 395-405.

Kabel, A. M. and F. H. Baali (2015). "Breast cancer: insights into risk factors, pathogenesis, diagnosis and management." J Cancer Res Treat 3(2): 28-33.

Kim, D. H., S. Sabour, U. N. Sagar, S. Adams and D. J. Whellan (2008). "Prevalence of hypovitaminosis D in cardiovascular diseases (from the National Health and Nutrition Examination Survey 2001 to 2004)." American Journal of Cardiology 102(11): 1540-1544.

Kim, H., Y.-H. Choi, S. J. Park, S. Y. Lee, S. J. Kim, I. Jou and K. H. Kook (2010). "Antifibrotic effect of Pirfenidone on orbital fibroblasts of patients with thyroid-associated ophthalmopathy by decreasing TIMP-1 and collagen levels." Investigative ophthalmology \& visual science 51(6): 3061-3066.

Kozono, S., K. Ohuchida, D. Eguchi, N. Ikenaga, K. Fujiwara, L. Cui, K. Mizumoto and M. Tanaka (2013). "Pirfenidone inhibits pancreatic cancer desmoplasia by regulating stellate cells." Cancer research.

Kumral, A., M. Soluk-Tekkeşin, V. Olgaç, S. Doğru-Abbasoğlu, Ü. Türkoğlu and M. Uysal (2015). "Effect of olive leaf extract treatment on doxorubicin-induced cardiac, hepatic and renal toxicity in rats." Pathophysiology 22(2): 117-123.

Liu, Y. (2006). "Renal fibrosis: new insights into the pathogenesis and therapeutics." Kidney international 69(2): 213-217.

López-Novoa, J. M., C. Martínez-Salgado, A. B. 
Rodríguez-Peña and F. J. L. Hernández (2010).

"Common pathophysiological mechanisms of chronic kidney disease: therapeutic perspectives." Pharmacology \& therapeutics 128(1): 61-81.

Ma, Z., Y. Pan, W. Huang, Y. Yang, Z. Wang, Q. Li, Y. Zhao, X. Zhang and Z. Shen (2014). "Synthesis and biological evaluation of the pirfenidone derivatives as antifibrotic agents." Bioorganic \& medicinal chemistry letters 24(1): 220-223.

Macías-Barragán, J., A. Sandoval-Rodríguez, J. Navarro-Partida and J. Armendáriz-Borunda (2010). "The multifaceted role of pirfenidone and its novel targets." Fibrogenesis \& tissue repair 3(1): 16.

Mady, M. M. (2007). "Biophysical studies on collagen-lipid interaction." Journal of bioscience and bioengineering 104(2): 144-148.

Octavia, Y., C. G. Tocchetti, K. L. Gabrielson, S. Janssens, H. J. Crijns and A. L. Moens (2012). "Doxorubicin-induced cardiomyopathy: from molecular mechanisms to therapeutic strategies." Journal of molecular and cellular cardiology 52(6): 1213-1225.

Panichi, V., M. Migliori, D. Taccola, C. Filippi, L. De Nisco, L. Giovannini, R. Palla, C. Tetta and G. Camussi (2001). "Effects of 1, $25(\mathrm{OH}) 2 \mathrm{D} 3$ in experimental mesangial proliferative nephritis in rats." Kidney International 60(1): 87-95.

Park, J. W., J. W. Cho, S. Y. Joo, C. S. Kim, J. S. Choi, E. H. Bae, S. K. Ma, S. H. Kim, J. Lee and S. W. Kim (2012). "Paricalcitol prevents cisplatininduced renal injury by suppressing apoptosis and proliferation." European journal of pharmacology 683(1-3): 301-309.

Prud'Homme, G. J. (2007). "Pathobiology of transforming growth factor $\beta$ in cancer, fibrosis and immunologic disease, and therapeutic considerations." Laboratory investigation 87(11): 1077.
Rashikh, A., K. K. Pillai and A. K. Najmi (2014). "Protective effect of a direct renin inhibitor in acute murine model of cardiotoxicity and nephrotoxicity." Fundamental \& clinical pharmacology 28(5): 489-500.

Ricardo, S. D., H. Van Goor and A. A. Eddy (2008). "Macrophage diversity in renal injury and repair." The Journal of clinical investigation 118(11): 3522-3530.

Sanchez-Niño, M.-D., M. Bozic, E. CórdobaLanús, P. Valcheva, O. Gracia, M. Ibarz, E. Fernandez, J. F. Navarro-Gonzalez, A. Ortiz and J. M. Valdivielso (2011). "Beyond proteinuria: VDR activation reduces renal inflammation in experimental diabetic nephropathy." American Journal of Physiology-Renal Physiology 302(6): F647-F657.

Schaefer, C., D. Ruhrmund, L. Pan, S. Seiwert and K. Kossen (2011). "Antifibrotic activities of pirfenidone in animal models." European Respiratory Review 20(120): 85-97.

Schwarz, U., K. Amann, S. R. Orth, A. Simonaviciene, S. Wessels and E. Ritz (1998). "Effect of 1, 25 (OH) 2 vitamin D3 on glomerulosclerosis in subtotally nephrectomized rats." Kidney International 53(6): 1696-1705.

Wang, Y., Y. P. Wang, Y.-C. Tay and D. C. Harris (2000). "Progressive adriamycin nephropathy in mice: sequence of histologic and immunohistochemical events." Kidney International 58(4): 1797-1804.

Zittermann, A., S. Frisch, H. K. Berthold, C. Götting, J. Kuhn, K. Kleesiek, P. Stehle, H. Koertke and R. Koerfer (2009). "Vitamin D supplementation enhances the beneficial effects of weight loss on cardiovascular disease risk markers." The American journal of clinical nutrition 89(5): 1321-1327. 